\title{
Riesgo de oídio (Erysiphe necator) de la vid en relación con el desarrollo de los racimos
}

\author{
Paul Campbell, Carlos Bendek, Bernardo A. Latorre ${ }^{1}$ \\ Facultad de Agronomía e Ingeniería Forestal, Pontificia Universidad Católica de Chile. \\ Casilla 306-22, Santiago, Chile
}

\begin{abstract}
P. Campbell, C. Bendek, B.A. Latorre. 2007. Risk of powdery mildew (Erysiphe necator) outbreaks on grapevines in relation to cluster development. Cien. Inv. Agr. 34(1):5-11. Recently, severe and very destructive outbreaks of powdery mildew (Erysiphe necator) have occurred on grapevines (Vitis vinifera) in central and northern Chile. Powdery mildew epidemics may develop from inoculum surviving as mycelia on buds infected the previous season and/or as cleistothecia surviving on leaves and bark. Cluster susceptibility changes throughout the growing season, with young berries being highly susceptible and mature berries being relatively resistant. The risk of infection can be predicted based on the maximum air temperature. Aiming to improve the efficacy of powdery mildew control, this research was performed to study the risk of powdery mildew outbreaks in relation to grapevine cluster development. Our results demonstrated that fungicide spray application (kresoxim-methyl, $65-70 \mathrm{mg} \cdot \mathrm{L}^{-1}$ or myclobutanil, $24 \mathrm{mg} \cdot \mathrm{L}^{-1}$ ) at full flowering (stage 23) provided the best control of cluster powdery mildew on grapevine cvs. 'Criolla', 'Pedro Jimenez' and 'Semillón'. In contrast, disease incidence and severity invariably increased on clusters sprayed at other grapevine stages. This was related to the presence of highly favorable temperature conditions prevailing during flowering with moderate to high infection risk indexes. In conclusion, grapevine stages occurring between the beginning of flowering and the appearance of small berries (stages 19 through 29) were the most critical stages for powdery mildew development. Therefore, fungicide treatments applied during flowering appeared to be essential to control grapevine powdery mildew.
\end{abstract}

Key words: Disease forecasting, Erysiphe, fungicides, grapevine diseases, Uncinula necator.

\section{Introducción}

El oídio, causado por Erysiphe necator Schwein. (sin. Uncinula necator (Schwein.) Burril), es la enfermedad más importante de la vid (Vitis vinifera $\mathrm{L}$.) en la zona central y norte de Chile (Bendek et al., 2002; Riveros, 2000; Latorre, 1984).

El oídio reduce los rendimientos y la calidad de la uva de mesa. En uvas para vinificar, altera considerablemente las cualidades del mosto y del vino, al disminuir los sólidos solubles totales, la intensidad de color y aumentar la acidez total.

Recibido 12 de mayo 2006. Aceptado 08 de septiembre 2006

${ }^{1}$ Dirigir correspondencia a B.A. Latorre: blatorre@uc.cl
Del mismo modo, afecta negativamente las cualidades organolépticas del vino (Gadoury et al., 2001, Amati et al., 1996; Latorre et al., 1996).

Erysiphe necator persiste como micelio, asociado a yemas infectadas la temporada precedente, y/o como cleistotecios, los que se desarrollan en hojas y otros órganos aéreos de la vid. En zonas lluviosas es posible que las ascosporas constituyan la principal fuente de inóculo primario (Jarvis et al., 2002; Pearson and Gadoury, 1987); mientras el micelio asociado a yemas infectadas, aparentemente, es la principal fuente de inóculo primario en zonas extremadamente secas (Pearson and Gärtel, 1985; Sall y Wrysinski, 1982; Van Der Spuy y Mathee, 1977). 
La susceptibilidad de los racimos de la vid varía a través de la estación de crecimiento, siendo muy susceptibles entre cuaja y el envero (Gadoury et al., 2001). Sin embargo, el riesgo de infección depende de la presión de oídio en el viñedo, la que se relaciona muy directamente con la temperatura y humedad ambiental (Carroll and Wilcox, 2003). Actualmente, es posible pronosticar periodos de alto riesgo, tan sólo monitoreando la temperatura del aire en el viñedo (Jarvis et al., 2002). Con el propósito de mejorar la eficiencia de los tratamientos contra el oídio, se estudió el riesgo de infección bajo condiciones de campo en relación con el desarrollo estacional de los racimos de la vid.

\section{Materiales y métodos}

Información meteorológica e índice de riesgo de infección

La temperatura, humedad relativa y agua libre se obtuvieron en micro-estaciones meteorológicas (Campbell Scientific Inc., Logan UT, USA y Davis Instruments Corp., CA, USA), ubicadas en los respectivos viñedos. En cada lugar se registró la temperatura cada $15 \mathrm{~min}$, valores que se promediaron cada $60 \mathrm{~min}$.

En función de la temperatura máxima del aire, se estimó un índice de riesgo de infección (IRI). Según este índice, los periodos de infección comenzaron con 30 puntos al concurrir tres días consecutivos con al menos $6 \mathrm{~h}$ seguidas, con temperaturas máximas entre 20 y $30^{\circ} \mathrm{C}$. Posteriormente, se sumaron 20 puntos o se restaron 10 puntos por cada día en que se cumplió o no se cumplió, este requisito térmico, respectivamente. El índice IRI fluctuó entre 0 (sin riesgo de infección) y 100 puntos (muy alto riesgo de infección). Asimismo, diminuyó 10 puntos por día con temperatura máxima entre 30 y $35^{\circ} \mathrm{C}$ por $60 \mathrm{~min}$ o superior a $35^{\circ} \mathrm{C}$ por $15 \mathrm{~min}$. El índice IRI volvió a cero luego de tres días consecutivos sin satisfacer los requisitos térmicos (Gubler $e t$ al., 1999; Jarvis et al., 2002).

\section{Epocas críticas para el desarrollo del oídio}

En un primer ensayo realizado en un parrón de vid 'Criolla', altamente infectado con oídio la temporada precedente, ubicado en Santiago, se establecieron los siguientes tratamientos químicos. Estos se aplicaron al inicio de los periodos fenológicos críticos que se indican, de modo de mantener protegidos los racimos en los periodos siguientes: 1. Entre el estadio 12 (brotes con cinco hojas expandidas) y 17 (inflorescencias expuestas) (estadios según Eichhorn-Lorenz) (Pratt, 1988), 2. Entre los estadios 17 y 29 (bayas formadas con racimos comenzando a colgar) y 3 . Entre los estadios 29 y 35 (envero, inicio de la maduración de las bayas) (Cuadro 1). Todas las aplicaciones se realizaron aproximadamente cada 20 días con $65 \mathrm{mg} \cdot \mathrm{L}^{-1}$ de kresoxim-metil (Stroby 50 SC, BASF, Alemania), de modo de cubrir completamente cada racimo hasta producir un leve escurrimiento superficial.

Este experimento se repitió al año siguiente en viñedos cvs. 'Criolla', 'Semillón' y 'Pedro Jiménez', ubicados en Santiago, Calera de Tango y Ovalle, respectivamente. En Santiago y Calera de Tango se empleó $70 \mathrm{mg} \cdot \mathrm{L}^{-1}$ de kresoxim metil mientras que en Ovalle las plantas fueron tratadas con $24 \mathrm{mg} \cdot \mathrm{L}^{-1}$ de miclobutanil (Systhane 2 EC, Dow AgroSciences). Ambos fungicidas se emplearon con intervalos máximos de 20 días entre aplicaciones.

En Santiago y Calera de Tango, los racimos se trataron, una o más veces, al inicio de los periodos críticos siguientes: 1. Una aplicación en racimos expuestos (estadio 17) (estadios según Eichhorn-Lorenz) (Pratt, 1988). 2. Una aplicación a inicio de la floración, con menos del $10 \%$ de las caliptras caídas (estadio 19). 3. Una aplicación en plena floración, con aproximadamente el $50 \%$ de las flores abiertas (estadio 23). 4. Una aplicación con bayas de $7 \mathrm{~mm}$ de diámetro con racimos colgando (estadio 31). 5. Dos aplicaciones fungicidas realizadas en plena floración y cuando las bayas tuvieron aproximadamente $7 \mathrm{~mm}$ de diámetro (estadios 23 y 31, respectivamente). 6. Tres aplicaciones fungicidas realizadas a inicio de la floración, plena floración y con bayas de aproximadamente $7 \mathrm{~mm}$ de diámetro (estadios 19, 23 y 31, respectivamente). 7. Cuatro aplicaciones fungicidas realizadas en los estadios 17, 19, 23 y 31. 8. Testigo sin protección fungicida. En Ovalle, además de los tratamientos antes indicados, hubo un tratamiento adicional en floración temprana (estadio 21), con aproximadamente el $25 \%$ de 
Cuadro 1. Incidencia y severidad del oídio de la vid (Erysiphe necator) en racimos de vides tratadas químicamente en diferentes estadios fenológicos de la vid.

Table 1. Incidence and severity of powdery mildew (Erysiphe necator) obtained on clusters chemically treated on different stages of grapevine development.

\begin{tabular}{|c|c|c|c|c|}
\hline \multicolumn{3}{|c|}{ Plantas tratadas $(\mathrm{X})$ en los estadios siguientes: } & \multicolumn{2}{|c|}{ Oídio en racimos } \\
\hline 18 Oct & 8 Nov & 28 Nov & Incidencia & Severidad \\
\hline Estadios 12 & Estadios 17 & Estadios 29 & $\%$ & $\%$ \\
\hline $\mathrm{X}$ & & & $24 a b^{2}$ & $22 \mathrm{ab}^{2}$ \\
\hline $\mathrm{X}$ & $X$ & & 13 a & $31 \mathrm{ab}$ \\
\hline $\mathrm{X}$ & $\mathrm{X}$ & $\mathrm{X}$ & $12 \mathrm{a}$ & $15 \mathrm{a}$ \\
\hline \multirow[t]{2}{*}{$\mathrm{X}$} & $\mathrm{X}$ & & 8 a & 12 a \\
\hline & $\mathrm{X}$ & & $32 \mathrm{~b}$ & $42 \mathrm{ab}$ \\
\hline Testigo & & & $58 \mathrm{c}$ & $53 \mathrm{~b}$ \\
\hline
\end{tabular}

${ }^{1}$ Se usó $65 \mathrm{mg} \cdot \mathrm{L}^{-1}$ de kresoxim metil (Stroby $50 \mathrm{WG),} \mathrm{aplicados} \mathrm{cada} 20$ días aproximadamente, en los estadios 12 (brotes con cinco hojas), 17 (inflorescencias expuestas) y 29 (racimos colgando, bayas formadas). Estadios según Eichkorn-Lorenz (Pratt, 1988).

${ }^{2}$ Promedios de cuatro repeticiones seguidos por diferentes letras son estadísticamente diferentes entre sí según Duncan-Waller k ratio $t$ test $(\mathrm{p}=0,05)$.

${ }^{1}$ Plants were treated every 20 days with $65 \mathrm{mg} \cdot \mathrm{L}^{-1}$ de kresoxim methyl (Stroby $50 \mathrm{WG}$ ) at the following Eichhorn-Lorenz stages (Pratt, 1988): stage 12 (shoots with five leaves unfolded), stage 17 (clusters fully developed), stage 29 (the appearance of small berries with clusters beginning to hang).

${ }^{2}$ Means of four replicates followed by the same letters were not significantly different according to Duncan-Waller $k$ ratio $t$ test $(p=0.05)$.

las caliptras caídas (Cuadro 2).

Todos los tratamientos se realizaron con bomba manual orientada principalmente a cubrir los racimos hasta producir un leve escurrimiento superficial, empleando alrededor de $1000 \mathrm{~L} \cdot \mathrm{ha}^{-1}$. La importancia relativa de cada periodo crítico sobre el control de esta enfermedad se evaluó por medio de la incidencia y severidad del oídio desarrollado en los racimos después de 15 a 20 días del último tratamiento fungicida.

\section{Diseño y análisis estadísticos}

Los tratamientos experimentales se distribuyeron de acuerdo con un diseño de bloques completos al azar con cuatro repeticiones, empleando como unidad experimental un racimo por planta en Santiago y dos racimos en Calera de Tango y Ovalle. El efecto de cada tratamiento se evaluó por medio de la incidencia y severidad. La severidad se estimó por medio de una escala de 0 a 5 , en donde $0=$ baya sana y 1 a 5 bayas cubiertas con oídio de la forma siguiente: $1=1$ a $20 \%, 2=21$ a $40 \%$, $3=41$ a $60 \%, 4=61$ a $80 \%$ y $5=81$ a $100 \%$. Los resultados se sometieron a análisis de varianza y los promedios se separaron de acuerdo con Duncan-Waller k-ratio $t$ test $(\mathrm{p}<0,05)$. Los valores porcentuales se transformaronen $\sqrt{X+0,5}$ antes de realizar los análisis respectivos.

\section{Resultados y discusión}

De acuerdo con los resultados obtenidos, la época de aplicación de los tratamientos fungicidas tuvo un efecto significativo $(\mathrm{p}=0,01)$ sobre el control del oídio en los racimos, siendo crítico el periodo comprendido entre inicios de la floración y bayas de $7 \mathrm{~mm}$ de diámetro, aproximadamente (Cuadros 1 y 2).

En Santiago, hubo alta presión de oídio en 2001. Esto significó obtener 53 y $58 \%$ de severidad e incidencia de oídio en los testigos sin tratar, respectivamente (Cuadro 1). En estas condiciones, la incidencia disminuyó significativamente entre 8 y $13 \%$, en los tratamientos que incluyeron al menos una aplicación fungicida al inicio del estadio 17, correspondiente a inflorescencias expuestas $(08$ de noviembre). En función de la residualidad del fungicida kresoxim metil, es posible que la protección otorgada se haya extendido por alrededor de 20 días hasta bayas cuajadas, aproximadamente estadio 29. La incidencia y severidad del oídio disminuyó sólo parcialmente en racimos tratados en el estado de racimo expuesto (estadio 12) o en el estado de bayas recién formadas con racimos comenzando a colgar (estadio 29) (Cuadro 1). 
Cuadro 2. Efecto de los tratamientos fungicidas, sobre la incidencia y severidad del el oídio (Erysiphe necator), aplicados en diferentes estadios críticos del desarrollo estacional de la vid.

Table 2. The effect of fungicide treatment, applied during critical phenological periods of grapevine development, on the incidence and severity of powdery mildew (Erysiphe necator) outbreak.

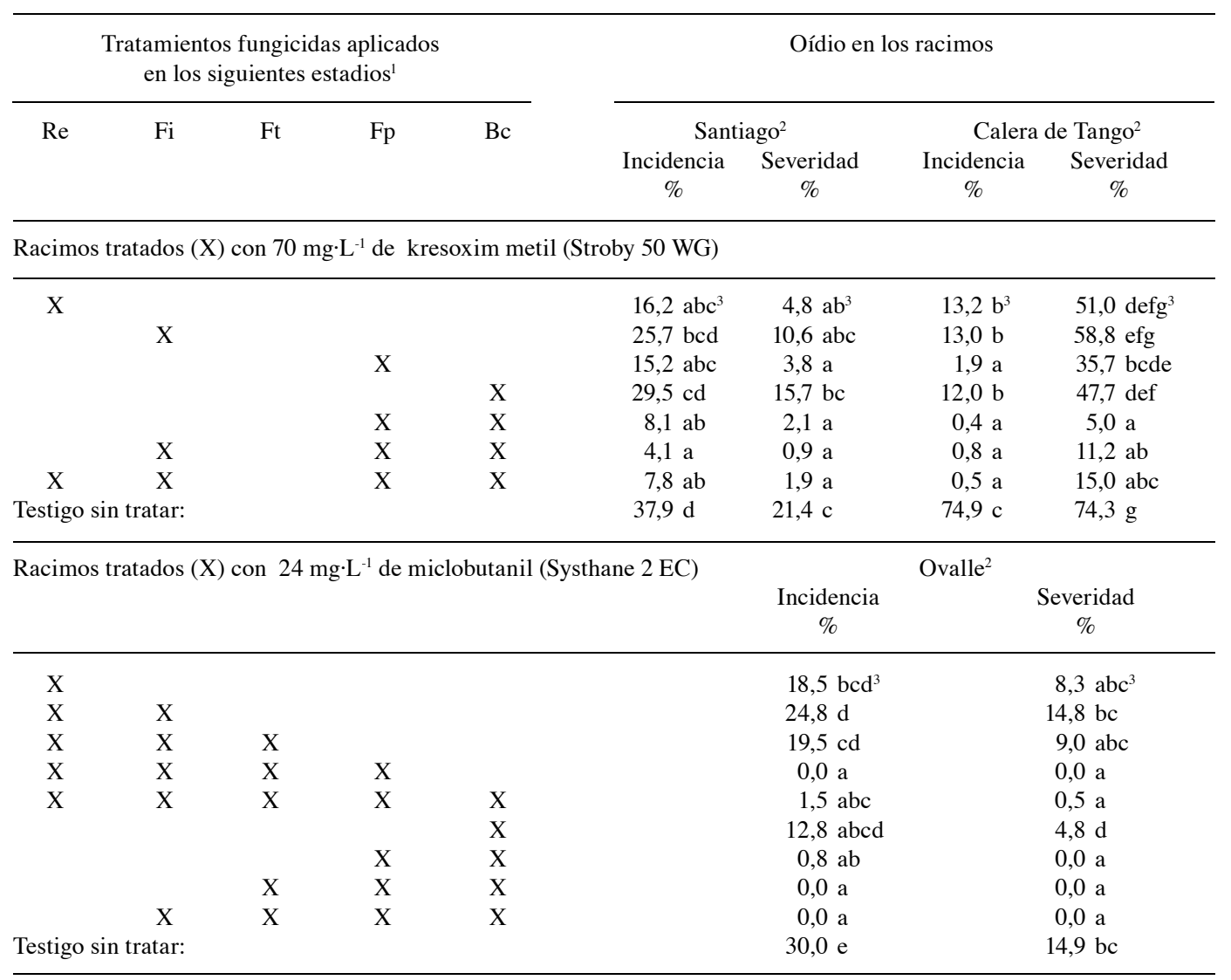

${ }^{1}$ Tratamientos fungicidas aplicados en los siguientes estadios fenológicos de la vid según Eichhorn-Lorenz (Pratt, 1988):

$\mathrm{Re}=$ Racimos expuestos (estadio 17), Fi= Inicios de la floración (estadio 19), Ft = Floración temprana 25\% de caliptras caídas, aproximadamente (estadio 21), $\mathrm{Fp}=$ Plena floración (estadio 23) y Bc= Bayas de $7 \mathrm{~mm}$ de diámetro (estadio 31).

${ }^{2}$ Vitis vinifera cvs. 'Criolla', 'Semillón' y Pedro Jiménez en Santiago, Calera de Tango y Ovalle, respectivamente.

${ }^{3}$ Promedios de cuatro repeticiones seguidos por diferentes letras son estadísticamente diferentes entre sí según Duncan-Waller k ratio $t$ test $(\mathrm{p}=0.05)$.

${ }^{\prime}$ Fungicide treatments were applied at the following Eichhorn-Lorenz stages (Pratt, 1988): Re $=$ Clusters fully developed (stage 17),

$F i=$ beginning of flowering (stage 19), Ft = early flowering with $25 \%$ cap fallen (stage 21), Fp = full flowering, 50\% cap fallen (stage 23) and $B c=$ Berries already formed with about $7 \mathrm{~mm}$ in diameter (stage 31).

${ }^{2}$ Vitis vinifera cvs. 'Criolla', 'Semillón' and Pedro Jiménez in Santiago, Calera de Tango and Ovalle, respectively.

${ }^{3}$ Means of four replicates followed by the same letters were not significantly different according to Duncan-Waller $k$ ratio t test $(p=0.05)$.

En Santiago en 2002, se obtuvo una moderada a alta presión de oídio en racimos sin tratar, con una incidencia de $37,9 \%$ y una severidad de $21,4 \%$. En estas condiciones, la época de los tratamientos fungicidas también tuvo un efecto significativo $(\mathrm{p}=0,01)$ sobre la protección de los racimos contra el desarrollo de oídio (Cuadro 2). Fue así como la incidencia $(4,1$ a $15,2 \%)$ y la severidad $(0,9$ a $3,8 \%)$ fueron considerablemente bajos en racimos tratados al inicio de plena floración (estadio 23), aun cuando desprotegidos en otros periodos (Cuadro 2). En forma similar, en racimos cv. Semillón en Calera de Tango en 2002, la presión de oídio fue alta, respectivamente 74,9 y $74,3 \%$ de incidencia y severidad, en los testigos sin tratar. En este viñedo, una sola aplicación fungicida en plena flor (estadio 23), realizada el 20 de noviembre, redujo la incidencia y severidad a 1,9 y $35,7 \%$, respectivamente. Sin embargo, la mayor protección contra oídio se obtuvo con una aplicación adicional realizada con bayas 
con $7 \mathrm{~mm}$ de diámetro (estadio 31) (Cuadro 2). En Ovalle el 2002, la mayor incidencia de oídiose obtuvo en el testigo sin tratar con un 30 y $14,9 \%$ de incidencia y severidad, respectivamente. Por el contrario, cinco tratamientos fungicidas realizados entre racimos expuestos (estado 17) y envero (estado 35) permitieron reducir significativamente $(\mathrm{p}=0,05)$ la incidencia y severidad del oídio. Sin embargo, es importante destacar que la desprotección de los racimos durante plena flor (estadio 23), invariablemente provocó un considerable incremento de incidencia y severidad de oídio en los racimos (Cuadro 2).

De acuerdo con las temperaturas máximas, condiciones moderadamente favorables al desarrollo del oídio (IRI $=40$ ) ocurrieron durante la brotación (Septiembre 23 y 24, 2002) en Santiago y Calera de Tango (la información de Ovalle no estuvo disponible) (Gubler et al., 1999; Jarvis et al., 2002). Por lo tanto, es posible que el inóculo haya aumentado inicialmente en brotes banderas, muy temprano en la temporada $\mathrm{y}$ mucho antes que se encontraran los primeros síntomas de oídio en el viñedo (Cuadro 3). Los brotes banderas se han considerado como una importante fuente de inóculo para el desarrollo de epidemias de oídio, particularmente en áreas donde este patógeno sobrevive como micelio latente en yemas infectadas (Rumbolz and Gubler, 2005; Cortesi, 2004; Haleen and Holz, 2001; Ypema and Gubler, 2000; Pearson and Gartel, 1985; Sall and Wrysinski,1982).

Indices de riesgo de infección bajos (IRI = 10) a moderados (IRI $=60$ ) se obtuvieron a inicio de la floración. Independientemente de la localización del viñedo, altos índices de riesgo de infección (IRI $=100$ ) ocurrieron en noviembre, durante plena floración de la vid. Posteriormente, las condiciones de temperatura se mantuvieron continuadamente favorables a la infección. Es interesante destacar que condiciones ambientales altamente favorables para la infección ocurrieron durante plena floración coincidiendo con el periodo más crítico para el control del oídio en éste y otros estudios (Silva, 2001; Riveros, 2000). Varios trabajos han demostrado que la resistencia ontogénica,

Cuadro 3. Indice de riesgo de infección de oídio (Erysiphe necator) estimados de acuerdo con las temperaturas máximas promedio del aire.

Table 3. Infection risk index for powdery mildew (Erysiphe necator) outbreak, estimated according to the mean of maximum air temperatures.

\begin{tabular}{|c|c|c|c|c|c|}
\hline & $\begin{array}{l}\text { Periodo de infección } \\
\text { fecha }\end{array}$ & $\begin{array}{l}\text { Temperatura } \\
\text { máxima }\end{array}$ & $\begin{array}{c}\text { Indice } \\
\text { de riesgo }\end{array}$ & $\begin{array}{l}\text { Riesgo de } \\
\text { infección }\end{array}$ & $\begin{array}{l}\text { Estadios } \\
\text { de la vid }{ }^{2}\end{array}$ \\
\hline $\mathrm{N}^{\circ}$ & inicio término & promedio, ${ }^{\circ} \mathrm{C}$ & 0 a 100 & & \\
\hline
\end{tabular}

\begin{tabular}{|c|c|c|c|c|c|c|}
\hline \multicolumn{7}{|c|}{ Santiago } \\
\hline 1 & $22-09$ & 27-09 & 15,8 & 40 & Moderado & Brotación \\
\hline 2 & $29-10$ & $02-11$ & 20,1 & 60 & Alto & Inicio floración \\
\hline 3 & $10-11$ & $29-11$ & 18,6 & 100 & Alto & Plena floración \\
\hline 4 & $01-12$ & $24-12$ & 19,8 & 100 & Alto & $\begin{array}{l}\text { Plena floración, bayas } \\
7 \mathrm{~mm} \text { de diámetro }\end{array}$ \\
\hline \multicolumn{7}{|c|}{ Calera de Tango } \\
\hline 1 & $23-09$ & 26-09 & 15,4 & 40 & Moderado & Brotación \\
\hline 2 & $29-10$ & $01-11$ & 16,4 & 10 & Leve & Inicio floración \\
\hline 3 & $10-11$ & $25-11$ & 18,1 & 100 & Alto & Plena floración \\
\hline 4 & $27-11$ & $10-01$ & 19,7 & 100 & Alto & $\begin{array}{l}\text { Plena floración, bayas } \\
7 \mathrm{~mm} \text { de diámetro }\end{array}$ \\
\hline
\end{tabular}

\footnotetext{
${ }^{1}$ Indice de riesgo de infección determinadas según Gubler et al. (1999), a partir de la temperatura promedio máxima del aire, registradas en micro estaciones meteorológicas ubicadas en cada viña.

${ }^{2}$ Estadios fenológicos de la vid (Vitis vinifera) según Eichhorn-Lorenz (Pratt, 1988).

${ }^{I}$ Infection risk was determined according to Gubler et al. (1999) on the basis of air temperatures that were monitored in micro weather stations placed in the vineyards.

${ }^{2}$ Stages of grapevine (Vitis vinifera) development according to Eichhorn-Lorenz (Pratt, 1988).
} 
desarrollada en bayas pequeñas, detiene el oídio en la medida que las bayas maduran, siendo posible que la infección ocurra muy temprano en el desarrollo de las bayas (Ficke et al., 2003; Gadoury et al., 2001). En parte, esto explicaría la eficacia relativamente baja obtenida con un sólo tratamiento fungicida, aplicado en racimos con bayas de $7 \mathrm{~mm}$ de diámetro (estado 31) pero previamente sin protección fungicida contra oídio de la vid.

En consecuencia, los resultados de este trabajo demostraron que condiciones favorables para el desarrollo de oídio ocurrieron durante la floración. Por lo tanto, el periodo comprendido entre inicios de la floración y bayas pequeñas (estadios 19 a 29) fue crítico para el desarrollo del ó́dio en los racimos. Tratamientos fungicidas en este periodo son indispensables para un buen control del oídio de la vid.

\section{Resumen}

En los últimos años, el oídio (Erysiphe necator) de la vid (Vitis vinifera) ha tenido especial importancia económica en la zona central y norte de Chile. Epidemias se desarrollan a partir del micelio latente que sobrevive en yemas infectadas la temporada precedente y/o a partir de cleistotecios desarrollados en hojas $u$ otros órganos infectados de la vid. La susceptibilidad de los racimos varia a través de la temporada, siendo muy susceptibles las bayas jóvenes y resistentes en la medida que maduran. El riesgo de infección se puede pronosticar en función de la temperatura máxima del aire. Con el propósito de mejorar la eficacia del control del oídio, se realizó este trabajo con el objetivo de estudiar el riesgo de infección en función del desarrollo del racimo. Los resultados obtenidos demostraron que las aplicaciones fungicidas (kresoxim-metil, $65-70 \mathrm{mg} \cdot \mathrm{L}^{-1}$ or miclobutanil, $\left.24 \mathrm{mg} \cdot \mathrm{L}^{-1}\right)$ aplicadas a inicios de plena floración (estadio 23) proporcionó el mejor control del oídio en los racimos de vides cvs. 'Criolla', 'Pedro Jimenez' y ‘Semillón'. Por el contrario, la incidencia y severidad invariablemente aumentó en racimos tratados en otros estadios. Esto se relacionó con la existencia de temperaturas del aire muy favorables al desarrollo de oídio, las que predominaron durante la floración, determinando índices de riesgo de infección moderados a altos. En conclusión, los estadios del desarrollo comprendidos entre inicios de la floración y bayas pequeñas (estadios 19 a 29) fue el periodo más crítico para el desarrollo del oídio. Por lo tanto, tratamientos fungicidas aplicados durante la floración son indispensables para controlar esta enfermedad.

Palabras clave: Erysiphe, enfermedades de la vid, fungicidas, pronóstico de enfermedades Uncinula necator.

\section{Agradecimientos}

Este trabajo se financió parcialmente gracias a los aportes recibidos de Fondecyt, projecto 1010542, de la Comisión Nacional de Investigación Científica y Tecnológica (CONICYT), Santiago, Chile.

\section{Literatura citada}

Amati, A., A. Piva, M. Castellari, and G. Arfelli. 1996. Preliminary studies on the effect of Oidium tuckeri on the phenolic composition of grapes and wines. Vitis 35:149-150.

Bendek, C., R. Torres, P. Campbell y B. Latorre. 2002. Aportes al conocimiento y control del oídio de la vid. Aconex (Chile) 76:5-11.

Carroll, J.E., and W.F. Wilcox. 2003. Effects of humidity on the development of grapevine powdery mildew. Phytopathology 93:11371144.

Cortesi, P., M.-P. Ottaviani, and M.G. Milgroom. 2004. Genetic analysis of a flag shoot subpopulation of Erysiphe necator in Italy. Phytopathology 94:544-550.

Ficke, A., D.M. Gadoury, R.C. Seem, and I.B. Dry. 2003. Effects of ontogenic resistance upon establishment and growth of Uncinula necator on grape berries. Phytopathology 93:556-563.

Gadoury, D.M., R.C. Seem, R.C. Pearson, W.F. Wilcox, and R.M. Dunst. 2001. Effects of powdery mildew on vine growth, yield and quality of Concord grapes. Plant Disease 85:137-140.

Gubler, W.D., M. Rademacher, S.J. Vasquez, and C.S. Thomas. 1999. Control of powdery mildew using the UC Davis powdery mildew risk index. APSnet Feature. Published online. The American Phytopathological Society, St. Paul, MN.

Haleen, F., and G. Holz, 2001. An overview of the biology, epidemiology and control of Uncinula necator (powdery mildew) on grapevine, with 
reference to South Africa. South African Journal for Enology and Viticulture 22:111-121.

Jarvis, W.R., W.D. Gubler, and G.G. Grove. 2002. Epidemiology of powdery mildews in agricultural pathosystems. Pages 169-199. In: Bólanger, R. R., W.R. Bushnell, A.J. Dikana and L.W. Timothy (eds.). The Powdery Mildews a Comprehensive Treatise. St. Paul. Minnesota. USA.

Latorre, B.A. 1984. Oídios de los árboles frutales y vides. Aconex (Chile) 7:5-9.

Latorre, B.A., Ph. Pszczólkowski, R. Torres y J.C. Broome. 1996. Efectividad de los ácidos grasos e inhibidores de esteroles contra el oídio de la vid y acción sobre la vinificación en Chile. Fitopatología 31:52-58.

Pearson, R.C., and D.M. Gadoury. 1987. Cleistothecia, the source of primary inoculum for grape powdery mildew in New York. Phytopathology 77:1509-1514.

Pearson, R.C., and W. Gartel. 1985. Occurrence of hyphae of Uncinula necator in buds of grapevine. Plant Disease 69:149-151.

Pratt, C. 1988. Grapevine structure and growth stages. In: Compendium of Grape Diseases. Pearson, R.C. and A.C. Goheen (eds.). The American Phytopathological Society, Paul, Minnesota, USA. p. 3-7.
Riveros, F. 2000. Control del oídio de la vid en la zona norte de Chile basado en la fenología de dos variedades de vid. Aconex (Chile) 67:2125.

Rumbolz, J., and W.D. Gubler 2005. Susceptibility of grapevine buds to infection by powdery mildew Erysiphe necator. Plant Pathology 54:535-548.

Sall, M.A. 1980. Epidemiology of grape powdery mildew: a model. Phytopathology 70:338-342.

Sall, M.A. and J. Wrysinski. 1982. Perennation of powdery mildew in buds of grapevine. Plant Disease 66:678-679.

Silva, R. 2001. Estimación de valores de EC50 para poblaciones de Oidium tuckeri (Berk) provenientes de la III y IV Regiones y evaluación de un modelo predictivo para el control de Oidium tuckeri (Berk). Tesis INACAP, Santiago, Chile. 63 pp.

Van Der Spuy, J.E., and F.N. Mathee. 1977. Overwintering of the oidium stage of Uncinula necator in the buds of the grapevine. Plant Disease 61:612-615.

Ypema, H.L., and W.D. Gubler. 2000. The distribution of early season grapevine shoots infected by Uncinula necator from year to year: A case study in two California Vineyards. Am. J. Enol. Vitic. 51:1-6. 